PREPARED FOR THE U.S. DEPARTMENT OF ENERGY, UNDER CONTRACT DE-AC02-76CH03073

PPPL-3922

PPPL-3922

UC-70

Landau Damping and Anomalous Skin Effect in Low-pressure Gas Discharges:

Self-consistent Treatment of Collisionless Heating

by

Igor D. Kaganovich, Oleg V. Polomarov, and Constantine E. Theodosiou

January 2004

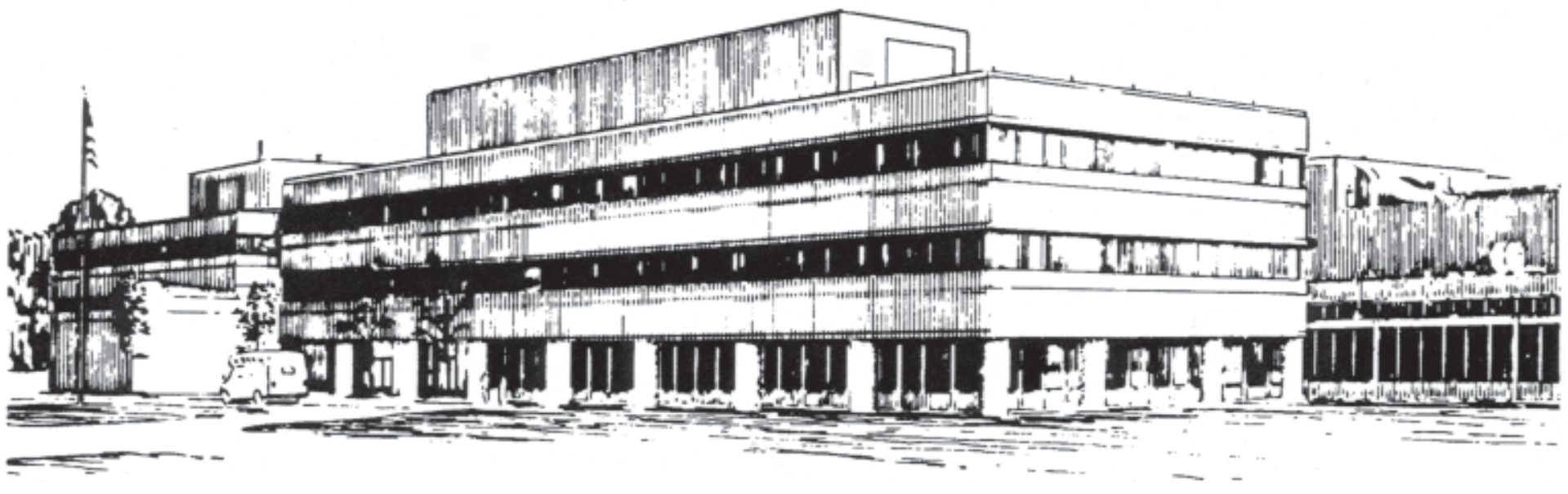

PRINCETON PLASMA PHYSICS LABORATORY PRINCETON UNIVERSITY, PRINCETON, NEW JERSEY 


\section{PPPL Reports Disclaimer}

This report was prepared as an account of work sponsored by an agency of the United States Government. Neither the United States Government nor any agency thereof, nor any of their employees, makes any warranty, express or implied, or assumes any legal liability or responsibility for the accuracy, completeness, or usefulness of any information, apparatus, product, or process disclosed, or represents that its use would not infringe privately owned rights. Reference herein to any specific commercial product, process, or service by trade name, trademark, manufacturer, or otherwise, does not necessarily constitute or imply its endorsement, recommendation, or favoring by the United States Government or any agency thereof. The views and opinions of authors expressed herein do not necessarily state or reflect those of the United States Government or any agency thereof.

\section{Availability}

This report is posted on the U.S. Department of Energy's Princeton Plasma Physics Laboratory Publications and Reports web site in Fiscal Year 2004. The home page for PPPL Reports and Publications is: http://www.pppl.gov/pub_report/

DOE and DOE Contractors can obtain copies of this report from:

U.S. Department of Energy

Office of Scientific and Technical Information

DOE Technical Information Services (DTIS)

P.O. Box 62

Oak Ridge, TN 37831

Telephone: (865) 576-8401

Fax: (865) 576-5728

Email: reports@adonis.osti.gov

This report is available to the general public from:

National Technical Information Service

U.S. Department of Commerce

5285 Port Royal Road

Springfield, VA 22161

Telephone: $1-800-553-6847$ or

(703) $605-6000$

Fax: (703) 321-8547

Internet: http://www.ntis.gov/ordering.htm 


\title{
Landau damping and anomalous skin effect in low-pressure gas discharges: self-consistent treatment of collisionless heating
}

\author{
Igor D. Kaganovich \\ Plasma Physics Laboratory, Princeton University, Princeton, NJ 08543 \\ Oleg V. Polomarov and Constantine E. Theodosiou \\ Department of Physics and Astronomy, \\ University of Toledo, Toledo, Ohio, 43606-3390.
}

(Dated: January 27, 2004)

\begin{abstract}
In low-pressure discharges, where the electron mean free path is larger or comparable with the discharge length, the electron dynamics is essentially nonlocal. Moreover, the electron energy distribution function (EEDF) deviates considerably from a Maxwellian. Therefore, an accurate kinetic description of the low-pressure discharges requires knowledge of the nonlocal conductivity operator and calculation of the non-Maxwellian EEDF. The previous treatments made use of simplifying assumptions: a uniform density profile and a Maxwellian EEDF. In the present study a self-consistent system of equations for the kinetic description of nonlocal, nonuniform, nearly collisionless plasmas of low-pressure discharges is reported. It consists of the nonlocal conductivity operator and the averaged kinetic equation for calculation of the non-Maxwellian EEDF. This system was applied to the calculation of collisionless heating in capacitively and inductively coupled plasmas. In particular, the importance of accounting for the nonuniform plasma density profile for computing the current density profile and the EEDF is demonstrated. The enhancement of collisionless heating due to the bounce resonance between the electron motion in the potential well and the external rf electric field is investigated. It is shown that a nonlinear and self-consistent treatment is necessary for the correct description of collisionless heating.
\end{abstract}




\section{INTRODUCTION}

Two basic phenomena: Landau damping and anomalous skin effect attract much interest in the plasma physics community. Both phenomena represent striking examples when the collisionless electron dynamics is considerably different from the collisional electron dynamics. The collisional electron dynamics is well described by Ohm's law - the electron current is proportional to the local electric field. If the electron mean free path is larger than the characteristic inhomogeneity scale of the electric field, an electron traverses significant distance between collisions and samples" different values of electric field along its way. As a result, the electron current is determined not by the local rf electric field, but rather is a function of the entire profile of the rf electric field (anomalous skin effect). Also for inhomogeneous electric fields another mechanism of heating or power dissipation, strikingly different from collisional one, is possible - the collisionless heating, that is determined by the wave-particle resonance and in most cases does not depend on the collision frequency (Landau damping).

The anomalous skin effect [1, 2] and Landau damping [3] were theoretically described in the late 40s. Further investigations were stimulated by fusion studies in late 60s and early 70 s, when a large number of theoretical and experimental papers elaborated on the details of both effects for plasmas (e.g., see reviews [4-6]). Recent resurgence of interest to the subject was invoked by applications of low-pressure discharges for plasma processing and lighting [7]. To increase fluxes of ions and radicals on a substrate, the semiconductor industry tends to use low-pressure gas discharges, typically with gas pressure of few milliTorrs. For these low pressures, it is easier to maintain plasma uniformity. Under these conditions the electron mean free path is large compared with the characteristic inhomogeneity scale of the electric field and the electron dynamics is collisionless. If one needs to understand power dissipation in such plasmas, the Landau damping and the anomalous skin effect have to be revisited for the conditions of low-pressure discharges.

Two major recent breakthroughs stimulated considerable advancement in the subject. Significant progress in refining the probe diagnostics of $\mathrm{rf}$ discharges has been achieved in the past decade [8]. That enabled experimentalists to measure detailed profiles of rf electric and magnetic fields, as well as the plasma potential and the electron energy distribution function (EEDF).

Significant progress has been also achieved in the simulation of rf discharges. The capa- 
bilities of modern computers allow to perform two- and three-dimensional simulations of gas discharges. Typically, they are particle-in-cell simulations with self- consistent electric and magnetic fields obtained from Maxwell's equations akin Ref. [9].

With experimental data and simulation results at hand, researchers may take on progressively more complicated situations. Research is advancing from one-dimensional, linear, non-self-consistent studies of uniform plasmas towards two- or three-dimensional, nonlinear kinetic self-consistent studies of nonuniform plasmas. This paper represents a compendium of recent results on the nonlinear kinetic and plasma self-organization.

\section{NONLINEAR LANDAU DAMPING}

In the rf electric field $E$, an electron oscillates back and forth with the rf velocity $\widetilde{v}$, according to

$$
m \frac{d \widetilde{\mathbf{v}}}{d t}=-e \mathbf{E}
$$

where $m$ and $e$ are the electron mass and charge, respectively. There is no heating on a long time scale in a steady-state operation. However, there is initial rf heating during switching on of the rf power [11]. The time-averaged electron kinetic energy increases due to oscillations because the kinetic energy is a nonlinear function of the velocity

$$
\left\langle\frac{m}{2}(v+\widetilde{v})^{2}\right\rangle=\frac{m v^{2}}{2}+\left\langle\frac{m \widetilde{v}^{2}}{2}\right\rangle>\frac{m v^{2}}{2} .
$$

There is collisionless heating also for the case of the localized rf field, when plasma electrons increase their kinetic energy on average due to resonant electron - wave interaction on the expenses of the rf wave energy and dissipate then the acquired energy in the bulk of the plasma. Inhomogeneity of the electric field is a key condition for collisionless heating [12]. This scenario may well describe the collisionless heating in rf discharges when the plasma size is much larger than the width of the wave penetration into the plasma. In the opposite case, the finite dimensions of the discharge have to be accounted for.

The collisionless electron heating by rf waves can be described by making use of the quasilinear theory [10]. In this theory the rf electric field is decomposed into a series of plane waves and the total heating is calculated as a sum of the electron heating by individual plane waves. Therefore, first it is important to thoroughly understand how collisionless heating 
occurs by a single plane wave. Here, we assume that the external electric field penetrates into a plasma and the wave amplitude is constant in time - continues operation of the discharge.

First, we analyze the electron heating in a non-self-consistent longitudinal rf wave with the rf electric field given by $E=E_{0} \cos (k x-\omega t+\phi)$ in an infinite plasma. The electron oscillation velocity of an electron moving with velocity $v_{x}$ calculated in the linear approximation $\left(v_{x}>>\right.$ $\left.\widetilde{v_{x}}\right)$ is given by

$$
\widetilde{v_{x}}=-\frac{e E_{0} \sin (k x-\omega t+\phi)}{m\left(\omega-k v_{x}\right)} .
$$

In the linear approximation $v_{x}=v_{x 0}$ is determined by the initial electron velocity on the right hand side of Eq. (2). Equation (2) has a singularity if the electron velocity equals the wave phase velocity $v_{x 0}=\omega / k$. To overtake the singularity problem, the further analysis of the resonant electrons has to account for the nonlinear effect: breaking the resonance condition $\omega=k v_{x}$ for the electrons moving with initial velocity $v_{x 0}$ due to the oscillatory velocity $v_{x}=v_{x 0}+\widetilde{v_{x}}$.

The nonlinear analysis is readily done in the frame of the wave. In this frame, electrons move in the electrostatic potential $\varphi(x)=-e \Phi_{0} \cos k x$, where $\Phi_{0}=E_{0} / k$. The evolution of the electron velocity is governed by the Hamiltonian

$$
H\left(v_{x}, x\right)=\frac{m}{2}\left(v_{x}-\frac{\omega}{k}\right)^{2}-e \Phi_{0} \cos k x .
$$

Electrons with total energy $H<e \Phi_{0}$ are trapped within the wave, as shown in Fig.1(the upper plot is drawn in dimensionless units). They perform nonlinear bouncing in the potential well with velocity $\widetilde{v}$ of order $\Delta u=\left(e \Phi_{0} / m\right)^{1 / 2}$. The linear theory of collisionless damping breaks down for times longer than the bounce time $\tau_{r} \equiv 1 / k \Delta u=\left(m / e \Phi_{0} k^{2}\right)^{1 / 2}$ of resonant electrons trapped in the potential well. In a steady state, the electron oscillatory velocity $\widetilde{v}$ lags in phase behind the rf electric field by $\pi / 2$ and the heating, being proportional to the time average $\langle\widetilde{v} E\rangle$, vanishes exactly. To break the strict correlation between $\widetilde{v}$ and $E$, some additional dynamic processes have to be accounted for. There can be, for example, collisions with walls or atoms. Collisions with walls may result in a dynamic chaos due to electron trajectory instability. The electron heating via dynamic chaos is described in the books [13] and [14]. Dynamic chaos requires special conditions on the amplitude and frequency of the wave. Here, we consider the opposite case where the dynamic chaos does not develop and collisions with atoms are responsible for the electron heating. To describe the collisionless heating we add rare collisions with gas atoms with frequency $\nu<<\omega$. From simulations 
and experimental data we know that the resulting collisionless heating does not depend on the electron collision frequency. However, as we discussed above, in the exact limit $\nu=0$ the collisionless heating vanishes. That begs the questions: (i) how is it possible, and (ii) whether there exist conditions where collisionless heating does depend on the collision frequency. To answer these questions we analyze the electron dynamics in the wave accounting for rare collisions.

Once again, for a single wave there is no heating without collisions because electrons acquire the energy from the wave during one half of the wave period and then return it during the other half. It sounds paradoxical, but it is necessary to account for rare collisions $\nu<<\omega$ to obtain net collisionless electron heating. In a partially ionized plasma, electrons collide mainly with neutral atoms. The differential cross section of electron-atom scattering is close to isotropic in the range of electron energies up to about $30 \mathrm{eV}[15,16]$. This means that during one collision the electron velocity rotates by a large angle and $v_{x}$ changes considerably. As a result, electrons leave the resonance region $\left|v_{x}-\frac{\omega}{k}\right| \sim \Delta u$ during one collision. This case is very different from the case of fully ionized plasmas where Coulomb collisions scattering dominates. In Coulomb collisions, the small angle scattering contributes most to the cross section. Therefore, for the case of fully ionized plasmas, electrons slowly diffuse out of the resonance region during many collisions, in contrast to the case of partially ionized plasmas, as it schematically shown in Fig.2.

The electron dynamics is a combination of bouncing in or near a potential well, then scattering out of the resonance region during a time of the order of the collision time $1 / \nu$, after which electrons experience many "fruitless" collisions during a time much longer than $1 / \nu$ until they accidently return back to the resonance region and so forth. Because of the relative simplicity of this collision process, it has been possible to solve analytically the Vlasov equation retaining both the nonlinear electron dynamics in the potential well and collisions accordingly, and to obtain the rate of collisionless electron heating [17]. The qualitative discussion of such a solution is given below.

Electron collisions with atoms are a random process. In between collisions electrons gain or loose energy from the wave as shown in Fig. 2. As a result of the combined action of many collisions and many interactions with the wave, electrons experience a diffusion in the energy space on time scales longer than the collision time and the wave period. The diffusion coefficient in energy space $D(\varepsilon)$ describes the formation of the electron energy distribution 
function $f_{0}(\varepsilon)$ (EEDF) and the collisionless heating. Indeed, when electrons diffuse in energy space, the average electron energy increases which corresponds to heating. Therefore, for the full description of the collisionless heating knowledge of $D(\varepsilon)$ is sufficient.

The diffusion coefficient in energy space $D(\varepsilon)$ is the product of the squared energy change $\Delta \varepsilon$ per step and the frequency of this step $\nu$, averaged over an electron ensemble with a given energy $\varepsilon$. In the linear approximation $\Delta \varepsilon=m v_{x} \widetilde{v}$, where $\widetilde{v}$ is given by Eq. (2) and the diffusion coefficient in energy space is

$$
D(\varepsilon)=\nu\left\langle(m \widetilde{\mathbf{v}} \cdot \mathbf{v})^{2}\right\rangle
$$

where the angular brackets denote averaging over the electron ensemble with a given energy $\varepsilon$.

Under typical discharge conditions, the elastic collision frequency is large compared to the inelastic collision frequency. Therefore, an electron experiences a lot of collisions prior to losing energy due to ionization or excitation. Elastic collisions scatter the electron velocity on a large pitch angle and make the electron energy distribution function isotropic. This means that in Eq. (4) the averaging has to be performed over all velocity directions.

Substituting $\widetilde{v_{x}}$ from Eq. (2) into Eq. (4) gives

$$
D(\varepsilon)=\frac{1}{2} \int \frac{d v_{x}}{2 v} \frac{\nu\left(e E_{0}\right)^{2} v_{x}^{2}}{\left(\omega-v_{x} k\right)^{2}}
$$

Here, $\varepsilon=m v^{2} / 2, \int d v_{x} / 2 v=\int d \cos \alpha d \beta / 4 \pi$ denotes averaging over velocity directions, and $1 / 2$ accounts for averaging over the phase of the wave. The integral in Eq. (5) diverges at the resonance $\omega=v_{x} k$; therefore, a more accurate description is necessary in the resonance region. The reason for divergence is the long time $1 /\left(\omega-v_{x} k\right)$ during which the electron stays in resonance with the electric field. There are two physical mechanisms which limit this time and destroy the resonance: One is collisions; if $\nu \sim\left(\omega-v_{x} k\right)$, an electron leaves the resonance region due to the collision and the amplitude of the velocity variation in Eq. (2) diminishes. The collisions are easy to account for by introducing an additional friction force into the electron motion equation (1). The resulting calculation requires the following substitution in Eq. (5)

$$
\frac{1}{\left(\omega-v_{x} k\right)^{2}} \rightarrow \frac{1}{\left(\omega-v_{x} k\right)^{2}+\nu^{2}}
$$

The other mechanism is nonlinear effects of the electron motion in the potential well. The linear estimate for $\widetilde{v_{x}}$ in Eq. (2) breaks down if $1 /\left(\omega-v_{x} k\right)>\tau_{r}$, i.e. for times longer than 
the bounce time of the trapped resonance electrons $\tau_{r}$. Then the velocity variation $\widetilde{v_{x}}$ is limited by $\Delta u=e E_{0} / m \tau_{r}$. Taking into account the nonlinear effects yields cumbersome calculations. However, the result is qualitatively similar to Eq. (6) with $\nu$ been replaced by $\tau_{r}^{-1}$. As a result of regularization of the resonance, Eq. (5) modifies to

$$
D(\varepsilon)=\frac{1}{2} \int_{-v}^{v} \frac{d v_{x}}{2 v}\left(e E_{0}\right)^{2} v_{x}^{2} \frac{\nu}{\left(\omega-v_{x} k\right)^{2}+\nu^{2}+\tau_{r}^{-2}} .
$$

In the limit $\nu \tau_{r}>>1$, the last factor on the right hand side of Eq. (7) can be simplified to

$$
\frac{\nu}{\left(\omega-v_{x} k\right)^{2}+\nu^{2}} \rightarrow \pi \delta\left(\omega-v_{x} k\right)
$$

where $\delta$ is the Dirac delta-function [10]. Indeed, the maximum of the resonance function in Eq. (8) is $\nu^{-1}$, whereas the width of the function is proportional to $\nu$. The integral does not depend on $\nu$. In this limit, $D(\varepsilon)$ is identical to the result of the quasilinear theory $D=D_{q l}$ and can be written as

$$
D_{q l}=\frac{\pi}{4} \frac{\left(e E_{0}\right)^{2}}{v k}\left(\frac{\omega}{k}\right)^{2} \Theta\left(v-\frac{\omega}{k}\right)
$$

where $\Theta$ is the Heaviside function. Electrons with velocity small compared to the wave phase velocity do not participate in the collisionless heating. Note that the diffusion coefficient is of the order of the square of the energy step $\left(e \Phi_{0}\right)^{2}$ multiplied by the frequency $\omega$ and does not contain any dependence on the collision frequency. In reality, diffusion in energy cannot occur without collisions, but because the width of the wave-particle resonance is proportional to $\nu$, the collision frequency disappears after integration of Eq. (7) in the limit $\nu \tau_{r}>>1$.

The role of the collision frequency is recovered by nonlinear analysis. In the opposite limit $\nu \tau_{r}<<1$, the last factor in Eq. (7) can be simplified to

$$
\frac{\nu}{\left(\omega-v_{x} k\right)^{2}+\tau_{r}^{-2}} \rightarrow \pi \nu \tau_{r} \delta\left(\omega-v_{x} k\right)
$$

Indeed, the maximum of the resonance function in Eq. (10) is $\nu \tau_{r}^{2}$ while the width of the function is proportional to $\tau_{r}^{-1}$, see Fig. 3. The integral diminishes in $\nu \tau_{r}^{2} \cdot \tau_{r}^{-1}=\nu \tau$ times compared to the previous case. As a result the diffusion coefficient in energy space is $\nu \tau$ times smaller than quasilinear estimate in Eq. (9), $D=\nu \tau D_{q l}$.

The exact analytical integration of the Vlasov equation accounting for nonlinear electron dynamics in a potential well and for collisions yields [17]

$$
D(\varepsilon) \cong D_{q l} \Pi\left(\nu \tau_{r}\right)
$$


The function $\Pi\left(\nu \tau_{r}\right)$ is given in Ref. [17] and plotted in Fig. 4. Surprisingly, this complicated function can be approximated within an error of less than $5 \%$ by $\tanh \left(2 \nu \tau_{r}\right)$. We can then deduce that the diffusion coefficient in energy space is related to the quasilinear result by

$$
D(\varepsilon) \cong D_{q l} * \tanh \left(2 \nu \tau_{r}\right)
$$

The above theory can be applied to the calculation of collisionless heating in a plasma. In the traditional theory collisionless heating is constant when the collision frequency tends to zero. In contrast to this, nonlinear effects cause the collisionless dissipation to tend to zero as $\nu$ approaches zero and to actually vanish in the limit $\nu=0$. The formula derived above gives the nonlinear wave damping for any value of the collision frequency. In the limit $\nu \tau_{r}>>1, \tanh \left(\nu \tau_{r}\right) \rightarrow 1$ and the obtained result coincides with the quasilinear theory. In the opposite case $\nu \tau_{r}<<1, \tanh \left(\nu \tau_{r}\right) \rightarrow 0$, and collisionless heating and corresponding wave damping vanishes, in accordance with the O'Neil theory [18]. Therefore, the result is a natural generalization of both theories, and gives the collisionless heating for any value of the collision frequency.

As can be seen from Fig. 4, the main contribution to heating at $\nu \tau_{r}>>1$ is due to electrons not trapped in the potential well of the wave. For $\nu \tau_{r}<<1, \Pi\left(\nu \tau_{r}\right) \cong 2 \nu \tau_{r}$ and collisionless heating is proportional to the collision frequency, similar to the result of Ref. [19]. Note that in contrast to Ref. [19], where only the limit of rare collisions was considered, Eq. (12) is valid for arbitrary values of $\nu \tau_{r}$. At small $\nu \tau_{r}$ the main contribution to the power dissipation is due to trapped-in-the-wave electrons; the contribution of untrapped electrons is only about $25 \%$ compared with that of trapped electrons. Note, that the obtained result is also very different from that of [19], where the nonlinear Landau damping with account for Coulomb collisions was explored. The main contribution to wave damping in that case is due to the narrow boundary layers around the separatrixes between trapped and untrapped electrons, see Fig. 1.

So far, we have considered electron heating by a monochromatic longitudinal wave. The theory can be applied to the calculation of collisionless heating in any bounded plasma for an arbitrary electric field. In many rf discharges, the electron plasma frequency is large compared to the discharge frequency. Therefore, the applied external rf electric field is screened near antennas or electrodes. An electron after passing the region of the rf electric field acquires a velocity kick, which consequently lead to electron heating. In the next section 
we consider collisionless heating with prescribed velocity kicks.

\section{COLLISIONLESS HEATING IN BOUNDED PLASMAS}

To demonstrate this concept we consider the simple example where the electron acquires a prescribed velocity kick $\Delta \mathbf{V} \cos \left(\omega t+\phi_{0}\right)$ near one wall and oscillates between two walls with separation $L$. Electrons experience collisions with frequency $\nu$ as in the previous section. A schematic of the electron dynamics is shown in Fig. 5.

\section{A. Influence of nonlinear effects on the diffusion coefficient in velocity space in}

\section{bounded plasmas}

We consider a transverse to the plasma boundary velocity kick $\Delta V_{y} \cos \left(\omega t+\phi_{0}\right)$ corresponding to model inductively coupled plasmas (ICP) in a slab geometry [6]. Collisions lead to a diffusion in the energy space. Similar to the case of collisionless heating by a single wave, the diffusion coefficient is the product of the squared energy change $\Delta \varepsilon$ and the collision frequency $\nu$, averaged over the electron ensemble with a given energy $\varepsilon$. In the linear approximation $\Delta \varepsilon=m v_{y} \widetilde{v_{y}}$, where $\widetilde{v_{y}}$ is the electron velocity change between collisions. The velocity variations $\widetilde{v_{y}}$ have an extreme maximum for resonant particles, which after bouncing between the walls during the time $2 L / v_{x}$ arrive at the left wall to acquire the same velocity kick, see Fig. 5. That requires the phase shift of the velocity kick to be equal to $2 \pi n$, where $n$ is an integer number. It's important to note ,that only resonant particles $\left(v_{x, n}=\omega L / \pi n\right)$ contribute to collisionless heating, similar to the case of longitudinal waves in the previous chapter, where only resonant particles $\left(v_{x}=\omega k\right)$ contribute to heating for unbounded plasmas. In the resonance region $\left(v_{x} \approx v_{x, n}\right)$, the evolution of the velocity is described by the system of equations [20]

$$
\frac{\Delta \widetilde{v_{y}}}{\Delta i}=\Delta V_{y} \sin \phi, \frac{\Delta \phi}{\Delta i}=\frac{\omega}{\Omega_{b}}-2 \pi n, \frac{\Delta t}{\Delta i}=\frac{1}{\Omega_{b}}
$$

where $\Delta$ denotes the change in a variable during one bounce, $\phi=\omega t-2 \pi n \Omega_{b} t+\phi_{0}-\pi / 2$ is the phase of the wave relative to the electron bounce motion, $i$ is a bounce number, $\Omega_{b}=v_{x} / 2 L$ is the bounce frequency, and $\Delta V_{y}$ is the amplitude of the kick. Near the resonance $\omega \approx 2 \pi n \Omega_{b}$ and the change in $\phi$ is small. Therefore, we can substitute the variations $\Delta$ in Eq. (13) by 
differentials. Combining the two first equations in (13) gives

$$
\frac{d \widetilde{v_{y}}}{d \phi}=-\frac{\Delta V_{y} \Omega_{b}}{\left(\omega-2 \pi n \Omega_{b}\right)} \sin \phi
$$

From Eq. (14) it follows that $\widetilde{v_{y}}$ oscillates near the resonance with amplitude $\Delta V_{y} \Omega_{b} /(\omega-$ $\left.2 \pi n \Omega_{b}\right)$. Note that

$$
N_{b}=\frac{1}{\omega / \Omega_{b}-2 \pi n}
$$

is the number of bounces an electron experiences before the phase $\phi$ changes to 1 and the kicks received by the electron have considerably different phase. Substituting $\widetilde{v_{y}}$ from Eq. (14) into Eq. (4) gives the diffusion coefficient in energy space

$$
D(\varepsilon)=\frac{m^{2} \Delta V_{y}^{2}}{2} \sum_{n=0}^{\infty} \int_{0}^{\pi} \frac{d \cos \alpha d \beta}{4 \pi} v_{y}^{2} \Omega_{b}^{2} \frac{\nu}{\left(\omega-2 \pi n \Omega_{b}\right)^{2}+\nu^{2}} .
$$

In the last factor of Eq. (16) we accounted for the fact that electrons leave the resonance region due to collisions and, consequently, the amplitude of velocity variation in Eq. (14) diminishes due to collisions. The rigorous derivation of the diffusion coefficient in energy space was performed in Ref. [10] making use of the quasi-linear theory. The result of the quasi-linear theory coincides with the euristic one-particle analysis of Ref. [20] discussed above.

If the effective width $\left(\delta v_{x}\right)$ of the resonant factor in Eq. (16) is much smaller than the thermal velocity $\left(\delta v_{x} \sim \nu L / \pi n<<V_{T}\right)$, the last factor of Eq. (16) can be replaced by a delta-function:

$$
D(\varepsilon)=\frac{m^{2} \Delta V_{y}^{2}}{2} \sum_{n=0}^{\infty} \int_{0}^{\pi} \frac{d \cos \alpha d \beta}{4 \pi}\left(v_{y}\right)^{2} \Omega_{b}^{2} \delta\left(\omega-2 \pi n \Omega_{b}\right) .
$$

As a result, the diffusion coefficient does not depend on the collision frequency.

The previous analysis, however, pertains to the non-self-consistent problem with given velocity kicks. In a real ICP plasma, the velocity kicks are directed along the $x$-axis, notwithstanding the fact that the rf electric field $E_{y}(x) \cos \left(\omega t+\phi_{0}-\pi / 2\right)$ is directed along the plasma boundary. It appears that the magnetic force $e \mathbf{v} \times \mathbf{B} / \mathbf{c}$ completely cancels the action of the electric field in the $y$-direction [21]. It can be readily explained invoking conservation of the canonical momentum $A_{y}$ due to symmetry in $y$-direction. The total canonical momentum $e A_{y} / c+m v_{y}$ is conserved. $A_{y}$ vanishes outside the skin layer where the rf field does not penetrate. Consider an electron starting the motion from the plasma 
bulk where $A_{y}=0$ and returning back. $A_{y}$ is unchanged after one pass through the skin layer. As a result, $v_{y}$ is unchanged also due to the conservation of the total momentum. This means that the action of the rf electric field and the rf magnetic field on the electron totaly compensate each other in the $y$-direction. The force $e v_{y} B_{z} / c$ generates the velocity kicks in the $x$-direction. The Lorentz force $e(\mathbf{E}+\mathbf{v} \times \mathbf{B} / c)$ produces the velocity kicks $\Delta V_{x}$ only in the direction transverse to the boundary [21]. It is a consequence of the momentum conservation - the momentum of the rf wave (photons) is imparted to electrons. The rf magnetic field rotates the velocity kick from the $y$ - to the $x$-direction, conserving the kinetic energy, i.e.

$$
V_{x} \Delta V_{x}=V_{y} \Delta V_{y}
$$

where $\Delta V_{y}$ is the velocity kick calculated taking into account the electric field only

$$
\Delta V_{y}=\frac{e}{m} \int E_{y}[x(\tau)] \cos \left(\omega \tau+\phi_{0}-\pi / 2\right) d \tau
$$

Here, the integral is taken along electron trajectory.

The fact that velocity kicks in ICP plasmas are directed in $x$-direction instead of $y$-direction have a profound impact on the collisionless heating at low collision frequencies. We saw in the previous section that nonlinear effects diminish the collisionless heating by a single longitudinal wave. In ICP, nonlinear effects are introduced by the fact that the bounce frequency itself depends on $v_{x}$. The velocity kicks change the bounce frequency and "kick" resonant electrons out of resonance.

This problem becomes similar to the nonlinear Landau damping problem, where nonlinear effects also destroy the resonance condition $\omega=v_{x} k$. In the resonance region, the evolution of velocity is described by a system of equations similar to Eq. (13), but where $\Omega_{b}$ is not constant and changes with $v_{x}$

$$
\frac{d v_{x}}{d i}=\Delta V_{x} \sin \phi, \frac{d \phi}{d i}=\frac{\omega}{\Omega_{b}}-2 \pi n
$$

Combining the two equations in Eq. (19) and using a Taylor series expansion near $v_{x, n}$ gives

$$
\frac{\omega}{\Omega_{b}\left(v_{x}\right)}-2 \pi n \approx-\frac{\omega}{\Omega_{b}} \frac{v_{x}-v_{x, n}}{v_{x, n}}
$$

and

$$
\frac{d v_{x}}{d \phi}=-\frac{\Delta V_{y} \Omega_{b} v_{x, n}}{\omega\left(v_{x}-v_{x, n}\right)} \sin \phi .
$$


Integrating Eq. (21) yields

$$
\left(v_{x}-v_{x, n}\right)^{2}-\frac{\Omega_{b} v_{x, n}}{\omega} \Delta V_{x} \cos \phi=\text { const. }
$$

Eq. (22) shows that even if the electron velocity initially satisfies the resonance condition $v_{x}=v_{x, n}$ exactly, the velocity perturbation $\widetilde{v_{x}}=v_{x}-v_{x, n}$ is limited by $\sqrt{\Omega_{b} v_{x, n} \Delta V_{x} / \omega}$. This is in contrast to Eq. (14). The number of bounces before the phase of the kick changes to 1 is given by Eq. (15). Substituting results of Eqs. (20) and (22) into Eq. (15) gives the typical time of changing of $\widetilde{v_{x}}$ near the resonance due to nonlinear effects

$$
\tau_{n l}=\frac{1}{\sqrt{\omega \Omega \Delta V_{x} / v_{x, n}}} .
$$

The system of equations (19) can be formally described by the Hamiltonian

$$
H\left(v_{x}, \varphi\right)=\frac{m}{2}\left(v_{x}-v_{x, r e s}\right)^{2}-\frac{m \Omega_{b} v_{x, n}}{2 \omega} \Delta V_{x} \cos \varphi
$$

This Hamiltonian coincides with the one in Eq. (3) of the electron trapped in a potential well. Thus, one can use here the results of the calculation of the diffusion coefficient for nonlinear Landau damping, Eq. (12), where time $\tau_{r}$ should be replaced by time $\tau_{n l}$. Therefore, the diffusion coefficient accounting for nonlinear effects reads

$$
D(\varepsilon)=\frac{m^{2} \Delta V_{y}^{2}}{2} \sum_{n=0}^{\infty} \int_{0}^{\pi} \frac{d \cos \alpha d \beta}{4 \pi}\left(v_{y}\right)^{2} \Omega_{b}^{2} \delta\left(\omega-2 \pi n \Omega_{b}\right) \tanh \left(2 \nu \tau_{n l}\right) .
$$

From Eq. (24) one can see that nonlinear effects are important at small $\nu$. In the limit $\nu \tau_{n l}<<1$, the diffusion coefficient is proportional to $\nu$ and $D(\varepsilon) \rightarrow 0$ as $\nu \rightarrow 0$. This is in contrast to the linear theory Eq. (17), where $D(\varepsilon)$ remains a constant as $\nu \rightarrow 0$.

A numerical example is shown in Fig. 6 for a model profile of the rf electric field $E_{y}(x)=$ $E_{0} \exp (-x / \delta)$. Fig. 6 is a plot of the diffusion coefficient for a fixed velocity $(v=5 \omega \delta)$ as a function of $\nu / \omega$, for two gap lengths $L=4 \delta / \pi$ and $25 \delta$. The diffusion coefficient was calculated by a Monte-Carlo method, as the ensemble averaged

$$
D_{v}=\left\langle\frac{1}{2} \frac{(\Delta v)^{2}}{\Delta t}\right\rangle
$$

where $\Delta v=v(t+\Delta t)-v(t)$ is the change in the absolute value of the velocity in a given period of time $\Delta t$. The Monte-Carlo simulation was compared with analytical results of the linear theory Eq. (16), and the nonlinear theory Eq. (24). The results presented in Fig. 6 
correspond to three values of the electric field. For small fields the velocity kicks are also small $\left(\Delta V_{x}<<v_{x}\right)$ and theory agrees well with the MC simulation. For larger values of the electric field the kicks are not small $\left(\Delta V_{x} \sim v_{x}\right)$ and the theory presented here is not applicable. The nonlinear effects with $\Delta V_{x} \sim v_{x}$ are also discussed in Ref. [23].

\section{B. Influence of nonlinear effects on the surface impedance in bounded plasmas}

In [22] and [24] the anomalous skin effect was considered ignoring the induced rf magnetic field. To show the importance of nonlinear effects discussed above, the real part of the surface impedance $Z$ with and without taking nonlinear effects into account was calculated. The real part of the surface impedance is related to the power absorption $P$ by $\operatorname{Re}(Z)=2 P|Z|^{2} / E_{0}^{2}$. The power deposition into a unit volume of plasma, $P$, can be expressed in terms of $D_{v}$ (Eq. (17) or Eq. (24) and the electron energy distribution function $f(\varepsilon)[10]$

$$
P=4 \pi \int_{0}^{\infty} v D(\varepsilon) \frac{d}{d \varepsilon} f(v) d \varepsilon
$$

Figure 7 depicts the real part of the surface impedance as a function of $\nu / \omega$. The profile of the electric field and the imaginary part of the surface impedance were taken in analytical form from [22]. This Figure shows that the value of the real part of the surface impedance decreases considerably at $\nu \tau_{n l}<1$ due to the influence of nonlinear effects. For typical values of electric field amplitudes in self-sustained ICP (about several V/cm [52]), the nonlinear effects start to be important for $\nu \sim 0.3 \omega$ and the difference with the linear theory can be as large as three orders of magnitude.

Until now, the collisionless heating was described only for a very simple case of a uniform plasma with a prescribed electric field, but for real discharges the electric field has to be determined self-consistently with EEDF and the ambipolar potential (or the density profile). This requirement significantly entangles the task of correct description of plasma heating. The way of handling of it is described in the next section. 


\section{SELF-CONSISTENT SYSTEM OF EQUATIONS FOR A KINETIC DESCRIP- TION OF THE LOW-PRESSURE DISCHARGES ACCOUNTING FOR THE NON- LOCAL AND COLLISIONLESS ELECTRON DYNAMICS}

We apply developed theory of collisionless heating to low pressure radio-frequency discharges. These discharges are extensively utilized for plasma processing and lighting [7]. Simulation of discharge properties is a common engineering tool for optimization of the plasma devices. Due to the large value of the electron mean free path $(\lambda)$ the electron current is determined not by the local rf electric field (Ohm's law), but rather is a function of the whole profile of the rf electric field over distances of order $\lambda$ (anomalous skin effect). Therefore, a rather complicated nonlocal conductivity operator has to be determined for the calculation of the rf electric field penetration into the plasma. Moreover, the electron energy distribution function (EEDF) is typically non-Maxwellian in these discharges [8]. The EEDF, nonlocal conductivity, and plasma density profiles are all nonlinear and nonlocally coupled. Hence, for accurate calculation of the discharge characteristics at low pressures, the EEDF needs to be computed self-consistently.

One of the ways to describe low-pressure discharge utilizes the so-called "nonlocal" approach. In the past, the nonlocal approach was based on the assumption that the electron mean free path is small compared with the discharge dimension. Recently we have updated this approach to include the collisionless phenomena like anomalous skin effect and landau damping into the description of nonlocal kinetics [61]. The nonlocal approach relies on the direct semi-analytic solution of the Boltzmann equation in the limiting regime where the electron energy relaxation length is much larger than the discharge gap [25-27]. Under these conditions the EVDF is almost isotropic and can be well approximated as a sum of the main isotropic part of EVDF $f_{0}$ and small anisotropic part of the EVDF $f_{1}$. Importantly, the main part of EVDF is a function of the total energy only $\left[f_{0}(\varepsilon)\right.$, where $\varepsilon=m v^{2} / 2-e \phi(\mathbf{r}), \phi(x)$ is the electrostatic potential], instead of being a function of velocities and spatial coordinates as in a general case $f_{0}(\mathbf{r}, \mathbf{v})$. This assumption allows significant simplifications of the Boltzmann equation, which effectively reduces from a six-dimensional (3D3V) problem in phase space to a $1 D$ problem for $f_{0}(\varepsilon)$ as a function of only $\varepsilon$. The final $1 \mathrm{D}$ equation for the electron energy distribution function is the temporal-spatial averaged Boltzmann equation over phase space available for the electron with a given total energy $\varepsilon$. The "nonlocal" approach 
is the opposite case to the "local" description of a plasma, where $f_{0}(\mathbf{r}, \mathbf{v})$ can be assumed to be a function of only the kinetic energy and the local rf electric field $f_{0}\left[m v^{2} / 2, \mathbf{E}(r)\right]$ whereas gradients of the local rf electric field and the influence of the ambipolar electric field are neglected. The nonlocal approach has been successfully applied to the self-consistent kinetic modelling of various low-pressure discharges: the capacitively coupled plasmas [28-31], the inductively coupled plasmas [32-35], the de discharges [36, 37], the afterglow [38], and the surface-wave discharges [39]. Additional references can be found in reviews [40-42].

If the gas pressure is less than 10mTorr, the electron mean free path becomes comparable or even larger than the discharge dimension and numerous collisionless phenomena dominate the discharge characteristics [6]. Therefore, wide utilization of low pressure discharges calls for "upgrading" of the nonlocal approach by taking into account collisionless phenomena. References [10] and [61] generalized the nonlocal approach for the low-pressure discharges to incorporate the collisionless heating and transit-time (electron temporal and spatial inertia) effects on the plasma conductivity in the discharge description. Here, we present the nonlocal approach with a rigorous, self-consistent treatment of collisionless phenomena in inhomogeneous plasmas. Similar approaches have been developed for calculation of the RF heating in tokamaks [46] and for the analysis of kinetic instabilities in intense beams [47].

The key assumption of the nonlocal approach is that the EEDF is isotropic and is a function of the total energy only. This assumption is based on the basic fact of atomic physics that for a typical electron energy of few electronvolts the elastic collision frequency is large compared to the inelastic collision frequency, which includes ionization and excitation of the background gas. An electron collides elastically many times with atoms before it losses energy due ionization or excitation. Elastic collisions scatter the electron velocity on a large pitch angle and make the EEDF isotropic. In between inelastic collisions, an electron acquires many velocity kicks from the rf electric field. This means that the electron thermal velocity is large compared with single velocity kick and quasilinear theory (diffusion in the energy space) can be utilized.

The derivations are lengthy. Therefore, and to be specific, the present analysis considers only the case of an inductively coupled plasma (ICP); the schematic of an ICP is shown in Fig. 8. Nevertheless, the approach has been designed in the most generalized way, so that derivations can be readily performed for other discharges. For example, in Ref. [31] the capacitive discharge, in Ref. [43] the electron-cyclotron-resonance discharge, and in Ref. [44] 
the surface-wave discharge were considered with self-consistent account for collisionless heating.

Most of the previously reported theoretical studies assume a uniform plasma, in a semiinfinite [45] or a slab geometry [48]. In this case the analytical treatment simplifies considerably, because electron trajectories are straight. In the semi-infinite geometry, electrons traverse the region of the rf electric field (skin layer) and are reflected back into the plasma at the discharge walls. An acquired velocity kick then dissipates in the plasma over distances of the order of the electron mean free path and subsequent kicks can be assumed independent. If the plasma dimension is small or comparable to $\lambda$, the subsequent kicks are correlated. The resonance between the wave frequency and the bounce frequency of the electron motion between walls may result in a modification of the nonlocal conductivity $[49,50]$ and may yield an enhanced electron heating [10, 20, 51]. The anomalous skin effect has been studied experimentally in cylindrical [49] and planar discharges [52]. Additional references can be found in the reviews of classic and recent works on the anomalous skin effect in gas discharge plasmas $[5,53]$. The theoretical studies in a cylindrical geometry are much more cumbersome, and have been done for uniform plasmas in Refs. [54-56] and for a parabolic potential well in Ref. [57]. Qualitative results in the cylindrical geometry are similar to the results in the plane geometry; therefore, in the present study only the one-dimensional slab geometry is considered.

For the case of a bounded uniform plasma, the electrostatic potential well is taken to be flat in the plasma and infinite at the wall (to simulate the existence of sheaths). In this square potential well, electrons are reflected back into the plasma only at the discharge walls. In a realistic non-uniform plasma, however, the position of the turning points will depend on the total (kinetic plus potential) electron energy and the actual shape of the potential well, i.e., low total energy electrons bounce back at locations within the plasma and may not reach regions of high electric field at all. As a result, the current density profiles in a nonuniform plasma may differ considerably from the profiles in a uniform plasma. The theory of the anomalous skin effect for an arbitrary electrostatic potential profile and a Maxwellian EEDF was developed by Meierovich and coworkers in Refs. [58-60] for the slab geometry. Although some rigorous analytical results of non-uniform plasmas have been reported, the detailed selfconsistent, nonlocal simulations related to such plasmas and comparison with experimental data are still lacking. Self-consistent, nonlocal simulations based on the approach developed 
in this paper were completed recently and presented in our separate publications [62, 63] and will be additionally reported in Ref.[64]. The alternative to the nonlocal approaches are based on particle-in-cell simulations, and only recently were capable of the detailed self-consistent, nonlocal kinetic simulations of low pressure discharges [9].

The kinetic description of the anomalous skin effect is similar to a well known mechanism of collisionless power dissipation - the Landau damping [3]. In an infinite plasma, the resonance particles that are moving with a velocity $\mathbf{v}$ close to the wave phase velocity, so that $\omega=\mathbf{v} \cdot \mathbf{k}$, interact intensively with wave fields. Therefore, the collisionless electron heating (and the real part of the surface impedance) depends on the magnitude of a Fourier harmonic of the electric field $E(k)$ and the number of the resonant particles $f\left(v_{x}=\omega / k\right)$, with $\mathbf{x} \| \mathbf{k}$. If the interaction with the skin layer is repeated in a resonant manner the momentum changes mount up. Therefore, the main contribution to the electron heating and the resistive part of the surface impedance comes from these near-resonant electrons. In a bounded plasma, the resonance condition requires that the bounce period $T_{b}$ be equal to one or several rf electric field periods: $T_{b}=2 \pi n / \omega$, where $n$ is an integer number. Because the bounce frequency depends on the electrostatic potential, accounting for the plasma nonuniformity is important for a correct calculation of the efficient power coupling.

The first unambiguous measurements of a bounce-resonance effect were performed in a non-neutral plasma. In Ref. [66] it was shown that the heating rate increases by a factor of $10^{4}$ as the oscillation frequency of the externally applied rf field is increased by a factor of 10 near the thermal electron bounce frequency.

As discussed above, the collisionless heating is determined by the number of resonant particles, and, hence, is dependent on the EEDF. The EEDF, in turn, is controlled by the collisionless heating. Only the particles which are in resonance with a wave are heated by the wave (collisionless heating). It means that in the regime of the collisionless dissipation, the form of the electron energy distribution function is sensitive to the wave spectrum. Therefore, a plateau in the EEDF can be formed in the regions of intensive collisionless heating, if the wave phase velocities are confined in some interval [65]. The evidence of a plateau formation for the capacitive discharge plasma were obtained in Ref. [51]. The cold electrons, which are trapped in the discharge center, do not reach periphery plasma regions where an intensive rf electric field is located and, as a result, these electrons are not heated by the rf electric field. The coupling between the EEDF shape and collisionless heating may 
result in a new nonlinear phenomenon: an explosive generation of the cold electrons [28]. The experimental evidence of the influence of collisionless phenomena on the EEDF shape were obtained in Refs. [52, 67-70].

\section{A. Self-consistent system of equations}

The self-consistent system of equations for the kinetic description of low-pressure discharges accounting for nonlocal and collisionless electron dynamics contains the averaged kinetic equation for $f_{0}(\varepsilon)$, the Maxwell equation for the rf electric field, the quasineutrality condition for the electrostatic potential, and the ion density profile given by the fluid conservation equations for ion density and ion momentum.

1. The averaged kinetic equation for $f_{0}$ reads

$$
-\frac{d}{d \varepsilon}\left(D_{\varepsilon}+\overline{D_{e e}}\right) \frac{d f_{0}}{d \varepsilon}-\frac{d}{d \varepsilon}\left[\overline{V_{e e}}+\overline{V_{e l}}\right] f_{0}=\sum_{k}\left[\overline{\nu_{k}^{*}\left(w+\varepsilon_{k}^{*}\right) \frac{\sqrt{\left(w+\varepsilon_{k}^{*}\right)}}{\sqrt{w}}} f_{0}\left(\varepsilon+\varepsilon_{k}^{*}\right)-\overline{\nu_{k}^{*}} f_{0}\right]
$$

where the upper bar denotes averaging according to

$$
\begin{aligned}
\overline{L(x, \mathbf{v})}(\varepsilon) & =\int_{x_{-}}^{x_{+}} d x v(x, \varepsilon) L[x, v(x, \varepsilon)], \\
v(x, \varepsilon) & =\sqrt{2[\varepsilon-\varphi(x)] / m}
\end{aligned}
$$

and the coefficients $V_{e l}, V_{e e}, D_{e e}$ are given by [71-73]

$$
\begin{gathered}
V_{e l}=\frac{2 m}{M} w \nu \\
V_{e e}=\frac{2 w \nu_{e e}}{n}\left(\int_{0}^{w} d w \sqrt{w} f\right) \\
D_{e e}=\frac{4}{3} \frac{w \nu_{e e}}{n}\left(\int_{0}^{w} d w w^{3 / 2} f+w^{3 / 2} \int_{w}^{\infty} d w f,\right) \\
\nu_{e e}=\frac{4 \pi \Lambda_{e e} e^{4} n}{m^{2} v^{3}}
\end{gathered}
$$

where $\nu_{e e}$ is the Coulomb collision frequency and $\Lambda_{e e}$ is the Coulomb logarithm. Note that at large electron energies $\varepsilon>>T_{e}, V_{e e} \approx 2 w \nu_{e e}$ and $D_{e e} \approx 2 w T_{e} \nu_{e e}$, where $T_{e}=$ $2 / 3 \int_{0}^{w} d w w^{3 / 2} f / n ; D_{\varepsilon}$ is given by $[10]$ 


$$
D_{\varepsilon}(\varepsilon)=\frac{\pi e^{2}}{4 m^{2}} \sum_{n=-\infty}^{\infty} \int_{0}^{\varepsilon} d \varepsilon_{x}\left|E_{y n}\left(\varepsilon_{x}\right)\right|^{2} \frac{\varepsilon-\varepsilon_{x}}{\Omega_{b}\left(\varepsilon_{x}\right)} \frac{\nu}{\left[\Omega_{b}\left(\varepsilon_{x}\right) n-\omega\right]^{2}+\nu^{2}},
$$

where

$$
E_{y n}\left(\varepsilon_{x}\right)=\frac{1}{\pi}\left[\int_{0}^{\pi} E_{y}(\theta) \cos (n \theta) d \theta\right] .
$$

Note that the above expression for $D_{\varepsilon}(\varepsilon)$ accounts for the bounce resonance $\Omega_{b}\left(\varepsilon_{x}\right) n=\omega$ and the transit time resonance $\omega=v / \delta$, which correspond to maxima of $E_{y n}\left(\varepsilon_{x}\right)$. Note also that if the discharge gap is increased to infinity the sum over $n$ can be replaced by integral and bounce resonances will be transformed into wave-particle resonances $\omega=k v$. The expression for $D_{\varepsilon}(\varepsilon)$ in Eq. (34) has the extra factor $v$ compared with $D(\varepsilon)$ in Eqs. (16). The $v$ was incorporated into expression of $D_{\varepsilon}(\varepsilon)$ for the correct account of the phase space volume on the right-hand side of the averaged kinetic equation, see Eq. (28).

2. The rf electric field is determined from the Maxwell equations

$$
\frac{d^{2} E_{y}}{d x^{2}}+\frac{\omega^{2}}{c^{2}} E_{y}=-\frac{4 \pi i \omega}{c^{2}}\left[j(x)+I \delta(x)-\delta_{\mathrm{anti}} I \delta(x-L)\right] .
$$

Here, $I$ is the current in the coil at $x=0 ; \delta_{\text {anti }}=0$, if there is the grounded electrode and no coil with current located at $x=L$ as in Fig. 8 , and $\delta_{\text {anti }}=1$, if there is a coil with the current $-I$ at $x=L$. The electron current density is given by [61]

$$
j_{y}(x)=\int_{0}^{x} G\left(x, x^{\prime}\right) E_{y}\left(x^{\prime}\right) d x^{\prime}+\int_{x}^{L} G\left(x^{\prime}, x\right) E_{y}\left(x^{\prime}\right) d x^{\prime}
$$

where

$$
G\left(x, x^{\prime}\right)=\frac{1}{2} \frac{e^{2}}{\sqrt{2 m}} \int_{\max \left(\varphi, \varphi^{\prime}\right)}^{\infty} \frac{\cosh \Phi \cosh \left(\Phi_{+}-\Phi^{\prime}\right)}{\sinh \Phi_{+}} \frac{\Gamma(\varepsilon)}{\sqrt{\varepsilon-\varphi(x)} \sqrt{\varepsilon-\varphi\left(x^{\prime}\right)}} d \varepsilon
$$

where we introduced a new function $\Gamma(\varepsilon)$

$$
\Gamma(\varepsilon) \equiv \int_{\varepsilon}^{\infty} f_{0}(\varepsilon) d \varepsilon
$$

The 1D slab system of two currents flowing in opposite directions describes very well a cylindrical configuration with radius $R$, where a coil produces rf currents at both plasma boundaries $x=0$ and $x=2 R, R=L / 2[48,49]$. Eqs. (36) and (37) can be solved numerically using a finite difference scheme. There is a major difficulty with such an approach: straightforward computing of the complex Green's function in Eq. (38) is slow and time consuming [62]. A better approach is to solve the integro-differential Eq. (36) making use 
of a spectral method, where the electric field is represented as a sum of harmonic functions. A robust procedure to solve this equation by FFT method is described in Ref. [61].

3. The electrostatic potential is obtained using the quasineutrality condition

$$
n_{i}(x)=\int_{\varphi(x)}^{\infty} f_{0}(\varepsilon) \sqrt{\varepsilon-\varphi(x)} d \varepsilon,
$$

where $n_{i}(x)$ is the ion density profile given by a set of fluid conservation equations for ion density and ion momentum [62]. Eq. (40) is solved in the form of a differential equation [29]

$$
\frac{d \varphi}{d x}=-T_{e}^{\mathrm{scr}}(x) \frac{d \ln \left[n_{i}(x)\right]}{d x}
$$

where $T_{e}^{\mathrm{scr}}(x)$ is the electron screening temperature

$$
T_{e}^{\mathrm{scr}}(x)=\left[\frac{1}{2 n(x)} \int_{\varphi(x)}^{\infty} f_{0}(\varepsilon) \frac{d \varepsilon}{\sqrt{\varepsilon-\varphi(x)}}\right]^{-1} .
$$

4. The power deposition can be computed as

$$
P(x)=\frac{1}{2} \operatorname{Re}\left[E_{y}^{*}(x) j(x)\right] .
$$

Integrating over the discharge length, Eq. ((43) becomes

$$
P=-\sqrt{2 m} \int_{0}^{\infty} D_{\varepsilon}(\varepsilon) \frac{d f_{0}(\varepsilon)}{d \varepsilon} d \varepsilon
$$

This equation can be used as a consistency check.

Figure 9 shows the rf electric field profiles calculated for a bounded plasma in a slab geometry with and without the ambipolar potential $\varphi=-4(x / R-1)^{2}$ (in Volts) for two cases: 1) the electron density at the electrode $n(0)$ is equal to the electron density of the uniform plasma $\left.n_{0}, n(0)=n_{0}, 2\right)$ the electron density in the center $n(R)$ is equal to $n_{0}$, $n(R)=n_{0}$, respectively. From Fig.9 it can be seen that taking into account an ambipolar potential greatly alters the rf electric field profile.

Figure 10 shows comparisons between experimental data [52] and simulation predictions using the non-local model [62]. The Ar ICP chamber was $19.8 \mathrm{~cm}$ in (inside) diameter and $10.5 \mathrm{~cm}$ in length. The coil current driving frequency was 6.78 MHz. The EEDF was assumed a Maxwellian, with an electron temperature obtained experimentally [52]. The plasma density was set to the value measured experimentally [52]. The normalization factor $E_{0}$ for the rf field at the wall on the coil-side was obtained by matching the simulated integrated power deposition with the experimental value for the power. 
Figures 10 (a) and (b) show reasonable agreement between the predicted (using the nonlocal 1-D model) and measured RF field and the current density profiles. The field decreases to a minimum and then goes through a hump. The qualitative features are captured with the model, although the precise location of the minimum is not predicted well. This is due to the fact that the actual discharge is $2-\mathrm{D}$, while the model is only 1-D.

Many previous works considered the anomalous skin effect for uniform plasmas without taking into account the electrostatic potential. We performed numerical simulations with and without taking into account an ambipolar potential. The presence of an ambipolar electrostatic potential greatly affects the electron heating in several ways: First, the electrostatic potential confines low energy electrons to the center of the discharge plasma and these electrons cannot reach the region of the strong field near the walls. Second, the number of resonant electrons is larger if an electrostatic potential is taken into account. For example, in the case of a parabolic potential, the bounce frequency is the same for all trapped electrons. That means that if $\omega=\Omega_{b}$, all trapped electrons are in resonance with the wave and effectively absorb wave energy. Fig. 11 shows the plasma resistivity, or the real part of impedance $Z(\Omega)$. The calculations were performed for the following discharge parameters: electron temperature $T_{e}=5 \mathrm{eV}$, peak electron density at the center of the discharge $n_{e}=5 \times 10^{11} \mathrm{~cm}^{-3}$, the rf field frequency $\omega=8.52 \times 10^{7} \mathrm{~s}^{-1}$, and the electron transport frequency $\nu=10^{7} \mathrm{~s}^{-1}$. In Fig. 11 it is clearly seen that the presence of the ambipolar potential enhances the resistivity of the plasma. The most profound changes in resistivity are for the quadratic potential for a specific value of $L$, which satisfies $\omega=\Omega_{b}=(40 \mathrm{eV} / \mathrm{m})^{1 / 2} / L$. The obtained results show explicitly that not taking into account the ambipolar potential as it is often done for simplicity - can lead to large discrepancies (more than 100 percent), especially for the conditions corresponding to the bounce resonance. Further results of the fully self-consistent calculations are presented in Ref. [64].

\section{Acknowledgments}

This research was supported by the U.S. Department of Energy Office of Fusion Energy Sciences through a University Research Support Program and the University of Toledo. The authors gratefully acknowledge helpful discussions with R. Davidson, D. Economou, Badri Ramamurthi, E. Startsev and L.D. Tsendin. 
[1] A. B. Pippard, Proc. R. Soc. A 191, 385 (1947).

[2] G. E. H. Reuter and E.H. Sondheimer, Proc. R. Soc. A 195, 336 (1948).

[3] L.D. Landau, J. Phys. (USSR) 10, 25 (1946).

[4] B. B. Kadomtsev, Sov. Phys. Uspekhi, 11, 328 (1968).

[5] V. I. Kolobov, and D. J. Economou, Plasma Sources Sci. Technol. 6, 1 (1997).

[6] M A Lieberman and V.A. Godyak, IEEE Trans. Plasma Sci. 26, 955 (1998).

[7] M.A. Lieberman and A.J. Lichtenberg, Principles of Plasma Discharges and Materials Processing, John Wiley \& Sons Inc. (New York) 1994.

[8] V. A. Godyak, R. B. Piejak, and B.M. Alexandrovich, Plasma Sources Sci. Technol. 11, 525 (2002).

[9] V. Vasenkov and M.J. Kushner, Phys. Rev. E. 66, 066411 (2002).

[10] Yu. M. Aliev, I. D. Kaganovich and H. Schluter, Phys. Plasmas 4, 2413 (1997); and in more details Yu. M. Aliev, I. D. Kaganovich and H. Schluter, "Collisionless electron heating in RF gas discharges. I. Quasilinear theory" in U.Korsthagen and L. Tsendin (Eds.), Electron kinetics and Applications of glow discharges, NATO ASI Series B, Physics 367, (Plenum Press, New York and London, 1998) 257.

[11] E.M. Lifshitz and L.P. Pitaevskii, "Physical Kinetics", (Pergamon Press, Oxford 1981) p.368.

[12] D.D. Ryutov, Plasma Phys. Control. Fusion 41, A1 (1991).

[13] A.J. Lichtenberg and M.A. Lieberman. "Regular and chaotic dynamics" 2nd ed. (SpringerVerlag, New York, 1992).

[14] R.Z. Sagdeev, D.A. Usikov, G.M. Zaslavsky, "Nonlinear physics : from the pendulum to turbulence and chaos" (Harwood Academic Publishers, New York, 1988).

[15] H.R.J.Walters, Phys. Rep. 116, 1 (1984).

[16] M. Adibzadeh and C. E. Theodosiou, "Differential, total, and momentum transfer cross sections for elastic scattering of electrons by inert gas atoms," Atom. Data Nucl. Data Tables, submitted (2003).

[17] I. D. Kaganovich, Phys. Rev. Lett. 82, 327 (1999).

[18] O’Neil, Phys. Fluids 8, 2255 (1965).

[19] V.E. Zakharov and V.I. Karpman, Sov. Phys. JETP 16, 351 (1963). 
[20] I. D. Kaganovich, V. I. Kolobov and L. D. Tsendin, Appl. Phys. Lett. 69, 3818 (1996).

[21] R.H. Cohen and T.D. Ronglien, Plasma Sources Sci. Techn. 5, 442 (1996).

[22] K.C. Shaing and A.Y. Aydemir, Phys. Plasmas 4, 3166 (1997).

[23] Yu.O. Tyshetskiy, A.I. Smolyakov, and V.A. Godyak, Phys. Rev. Lett. 90, 255002 (2003); Yu.O. Tyshetskiy, Ph.D. Thesis, University of Saskatchewan (2003) http://library.usask.ca/theses/available/etd-12182003-161103.

[24] M.M. Turner, Phys. Rev. Lett. 71, 1844 (1993).

[25] I.B. Bernstein and T. Holstein, Phys. Rev. 94, 1475 (1954).

[26] L.D. Tsendin, Sov. Phys. JETP 39, 805 (1974).

[27] L.D. Tsendin and Yu. B. Golubovskii, Sov. Phys. Tech. Phys. 22, 1066 (1977).

[28] S. V. Berezhnoi, I. D. Kaganovich and L. D. Tsendin, Plasma Physics Reports 24, 556 (1998).

[29] S. V. Berezhnoi, I. D. Kaganovich and L. D. Tsendin, Plasma Sources Sci. Technol., 7, 268 (1998).

[30] S.V. Berezhnoi, I.D. Kaganovich, M. Mišina, A. Bogaerts and R. Gijbels, IEEE Transactions on plasma science $\mathbf{2 7}, 1339$ (1999).

[31] Igor D. Kaganovich, "Anomalous Capacitive Sheath with Deep Radio Frequency Electric Field Penetration," Phys. Rev. Lett. 89, 265006 (2002); and a fuller version in http://arxiv.org/PS_cache/physics/pdf/0203/0203042.pdf.

[32] V. I. Kolobov and W. N. G. Hitchon, Phys. Rev. E 52, 972 (1995).

[33] V. I. Kolobov, G.J. Parker and W. N. G. Hitchon, Phys. Rev. E 53, 1110 (1996).

[34] U. Kortshagen, I. Pukropski and L. D. Tsendin, Phys. Rev. E 51, 6063 (1995).

[35] G. Muemkin, J. Phys. D 32, 804 (1999).

[36] V. I. Kolobov, and L. D. Tsendin, Phys. Rev. E 46, 7837 (1992).

[37] Y. Benke and Y.B. Golubovskij, Sov.Phys.-Tech. Phys. 39, 38 (1994).

[38] R.R. Arslanbekov and A.A. Kudryavtsev, Phys. Rev. E 58, 7785 (1998).

[39] Yu. M. Aliev, H. Schlueter and A. Shivarova, "Guided-Wave-Produced Plasmas" (Springer, Berlin-Heidelberg-New York 2000), chapter 6.

[40] L. D. Tsendin, Plasma Sources Sci. Technol. 4, 200 (1995).

[41] V. I. Kolobov and V. A. Godyak, IEEE Trans. Plasma Sci 23, 503 (1995).

[42] U. Kortshagen, C. Busch and L. D. Tsendin, Plasma Sources Sci. Technol. 5, 1 (1996).

[43] I.D. Kaganovich, M. Mišina, R. Gijbels and S.V. Berezhnoi, Phys. Rev. E. 61, 1875 (2000). 
[44] Yu. M. Aliev, U. Kortshagen, A.V. Maximov, H. Schlueter and A. Shivarova, Phys. Rev. E. 51, 6091 (1995).

[45] E. S. Weibel, Phys. Fluids 10, 741 (1967).

[46] Dirk Van Eester, J. of Plasma Physics 65, 407 (2001).

[47] E. Startsev and R.C. Davidson, privite communication.

[48] H. A. Blevin, J. A. Reynolds, and P. C. Thonemann, Phys. of Fluids 13, 82 (1970).

[49] H. A. Blevin, J. A. Reynolds, and P. C. Thonemann, Phys. of Fluids 16, 82 (1973).

[50] K. C. Shaing, and A. Y. Aydemir, Phys. of Plasmas 4, 3163 (1997).

[51] U. Buddemeier, I. Kaganovich, "Collisionless electron heating in RF gas discharges. II. Role of collisions and non-linear effects", in U.Korsthagen and L. Tsendin (Eds.), "Electron kinetics and Applications of glow discharges", NATO ASI Series B, Physics 367(Plenum Press, New York and London, 1998) 283.

[52] V. A. Godyak and R. B. Piejak, J. Appl. Phys. 82, 5944 (1997).

[53] F. F. Chen, Phys. of Plasmas 8, 3008 (2001).

[54] B. E. Meierovich, Sov. Phys. JETP 10, 782 (1970).

[55] N. S. Yoon, S. S. Kim, C. S. Chang, and Duk-In Choi, Phys. Rev. E 54, 757 (1996).

[56] V. I. Kolobov, D.P. Lymberopoulos, and D. J. Economou, Phys. Rev. E. 55, 3408 (1997).

[57] R.G. Storer, Phys. of Fluids 16, 949 (1973).

[58] M. A. Liberman, B. E. Meierovich, and L. P. Pitaevskii, Sov. Phys. JETP 35, 904 (1972).

[59] S. M. Dikman and B. E. Meierovich, Sov. Phys. JETP 37, 835 (1973).

[60] A. N. Vasiliev and B. E. Meierovich, Sov. Phys. JETP 40, 865 (1974).

[61] I. D. Kaganovich and O. Polomarov, Phys. Rev. E 68, 026411 (2003).

[62] B. Ramamurthi, D. J. Economou, and I. D. Kaganovich, Plasma Sources Sci. Technol. 12, 170 (2002);

[63] B. Ramamurthi, D. J. Economou, and I. D. Kaganovich, Plasma Sources Sci. Technol. 12, $302(2002)$

[64] O. Polomarov, C. E. Theodosiou and I. D. Kaganovich, "The influence of the ambipolar potential on the parameters of the ICP discharges at the condition of the bounce resonance", submitted to Phys. Rev. E (2004).

[65] A.A. Vedenov, Theory of a Weakly Turbulent Plasma, in: M.A. Leontovich (ed.), Reviews of Plasma Physics vol. 3 (Consultants Bureau, New York-London, 1967). 
[66] B.P. Cluggish, J.R. Danielson, and C.F. Driscoll, Phys. Rev. Lett. 81, 353 (1998).

[67] V. A. Godyak and V. I. Kolobov, Phys. Rev. Lett. 81, 369 (1998).

[68] V. A. Godyak, B.M. Alexandrovich and V. I. Kolobov, Phys. Rev. E 64, 0264061 (2001).

[69] Chin Wook Chung, K.-I. You, S. H. Seo, S. S. Kim, and H. Y. Chang, Phys. of Plasmas, 8, $2992(2001)$.

[70] Chin Wook Chung, S. S. Kim, S. H. Seo and H. Y. Chang, J. Appl. Phys. 88, 1181 (2000).

[71] V.L. Ginzburg and A.V. Gurevich, Sov. Phys. Usp. 3, 115 (1960).

[72] I.D. Kaganovich and L.D. Tsendin, IEEE Trans. Plasma Sci 20, 66 (1992).

[73] I.D. Kaganovich and L.D. Tsendin, IEEE Trans. Plasma Sci 20, 86 (1992). 
Figure 1. The bottom figure shows phase portrait of the trapped (solid line) and untrapped (dotted line) electrons. The separatrix line is shown as dashed. The potential is shown on top.

Figure 2. Illustration of the difference between electron-ion (small angle) and electronatom (large angle) collisions. The top right figure shows the differential scattering cross section. The bottom figure shows the electron velocity as function of time during several collisions.

Figure 3. Resonance function in Eq. (7) in the limits $\nu \tau_{r}>>1$ and $\nu \tau_{r}<<1$.

Figure 4. Dimensionless function $\Pi(\nu)$.

Figure 5. A schematic of the electron dynamics in a bounded plasma.

Figure 6. Influence of the second boundary on collisionless heating. Dimensionless diffusion coefficient in velocity space $G=D_{v} 2 m^{2} \omega L / e^{2} E_{0}^{2} \delta$ for an electric field $E_{y}=$ $E_{0} \exp (-x / \delta)$ as a function of $\nu / \omega$ for two different slab widths $L=4 \delta / \pi$ and $L=25 \delta$. Solid curves with circles correspond to the analytical formulae $(17,24)$, dashed lines are Monte Carlo simulations.

Figure 7. The real part of the surface impedance in Ohm, $\zeta$, as a function of $\nu / \omega$. The plasma parameters are: density $N=10^{11} \mathrm{~cm}^{-3}, T_{e}=5 \mathrm{eV}, L=4 \mathrm{~cm}$.

Figure 8. Schematic of an inductively coupled plasma. The antenna on the left produces inductive rf electric field (shown by a dashed line), which penetrates into the plasma over a distance of the order of the skin depth. Electrons are confined by the stationary electrostatic potential $\varphi(x)$. Electrons with different total energy $\varepsilon$ are confined in different regions of the plasma.

Figure 9. The profile of the normalized amplitude of the rf electric field calculated for the case of cylindrical-like geometry (slab geometry and two antisymmetric currents at $x=0$ and $x=2 R$ ) as a function of the normalized coordinate $x / R$. The electron temperature is $T_{e}=2.5 \mathrm{eV}$ and the uniform spatial electron density is $n_{0}=10^{12} \mathrm{~cm}^{-3}$, which corresponds to $\omega / \Omega_{b T}=1.5, \nu / \Omega_{b T}=0.3$, and $R \omega_{p} / c=4.5$. The solid line shows the profile for a uniform plasma. Symbols correspond to a nonuniform plasma $n(x)$ with a Boltzmann distribution and the ambipolar potential $\phi=-4(x / R-1)^{2}$ (in Volts) for two cases: 1) the electron density at the electrode $n(0)$ is equal to the electron density of the uniform plasma $n_{0}$, and in the rest of the plasma $n(x)>n_{0}$, and 2) the electron density $n(R)$ at the center is equal to $n_{0}$, and in the rest of the plasma $n(x)<n_{0}$. 
Figure 10. Comparison between experimental data [52] and simulation predictions using a non-local model: (a) RF electric field and (b) current density profiles, for a pressure of 1 mTorr.

Figure 11. Surface impedance as a function of discharge length for different plasma profiles. Plasma and discharge parameters are $n_{e}=5 \times 10^{11} \mathrm{~cm}^{-3}, f=13.56 \mathrm{MHz}, \nu=$ $10^{7} \mathrm{~s}^{-1}$, EEDF is Maxwellian with $T_{e}=5 \mathrm{eV}$. The solid line shows the impedance of a uniform plasma without any potential, the dashed line shows the impedance of a nonuniform plasma with a potential $\varphi(x)=-5(2 x / L-1)^{2}$ in $\mathrm{V}$, and the dash-dot line corresponds to a potential $\varphi(x)=-5(2 x / L-1)^{4}$. 

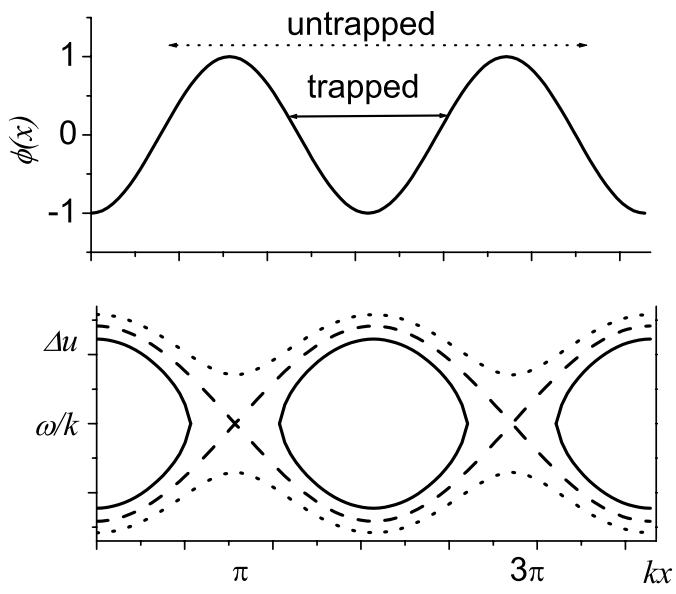

FIG. 1:

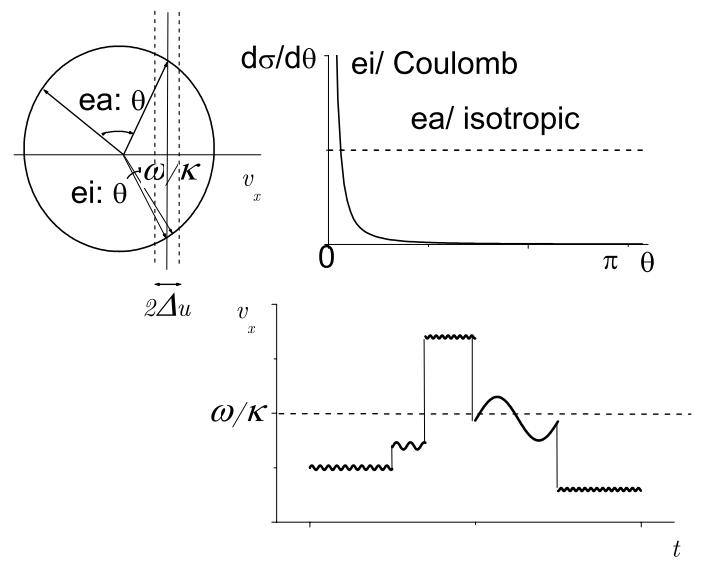

FIG. 2: 


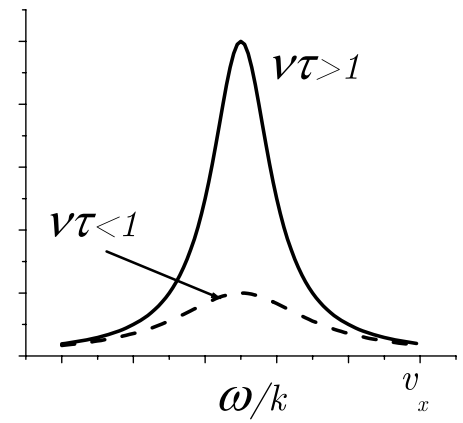

FIG. 3:

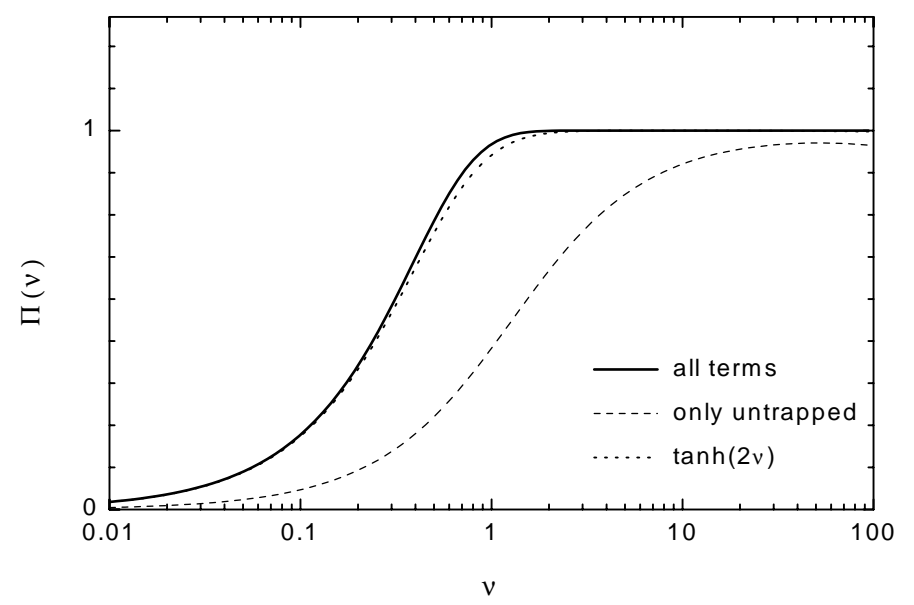

FIG. 4: 


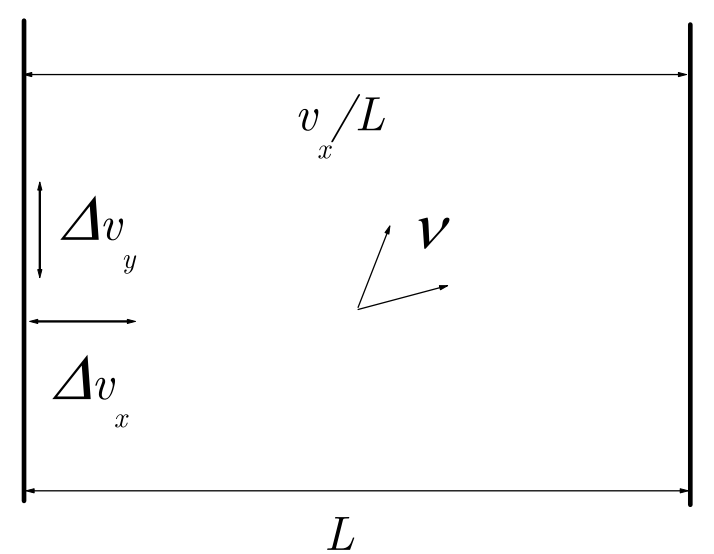

FIG. 5:

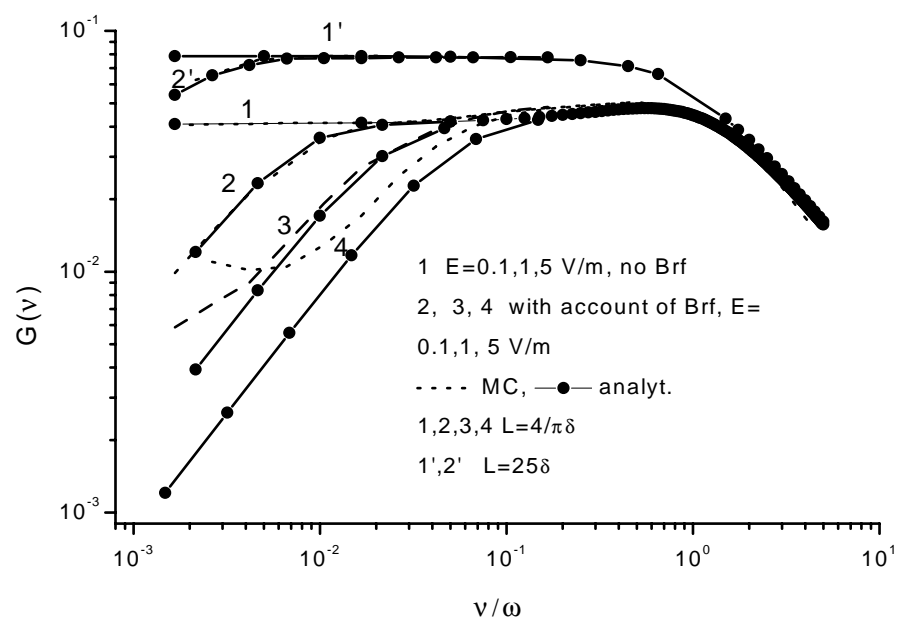

FIG. 6: 


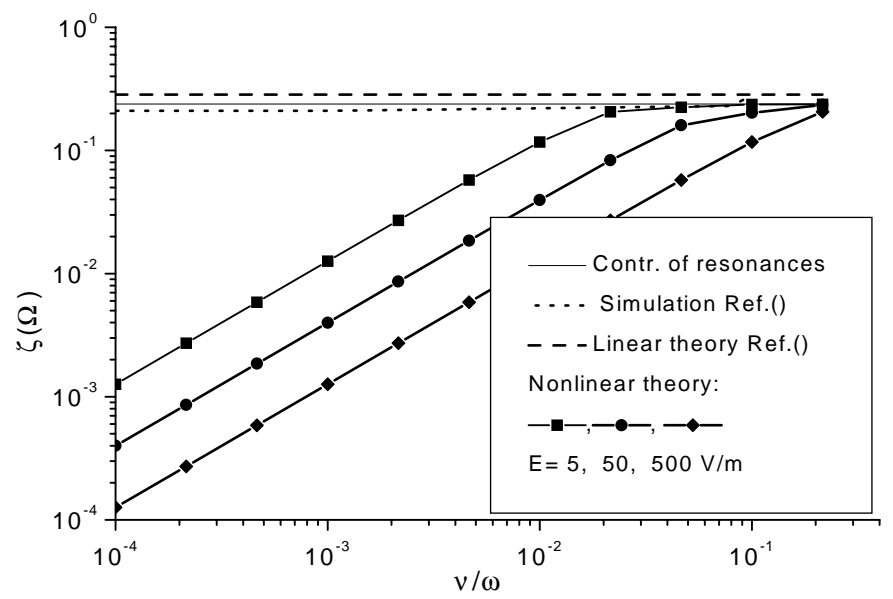

FIG. 7:

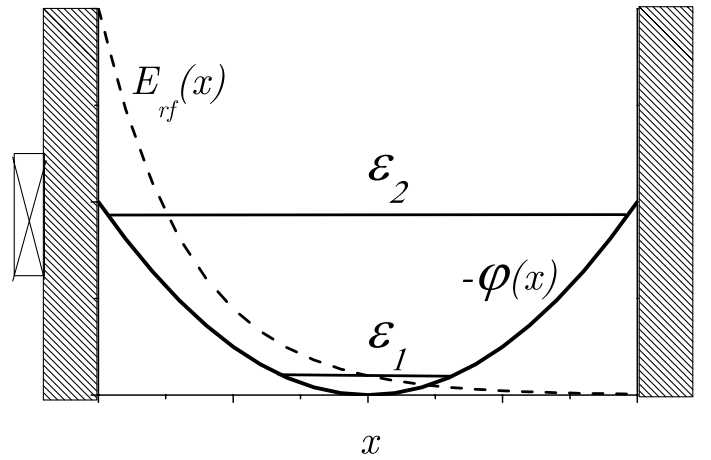

FIG. 8: 


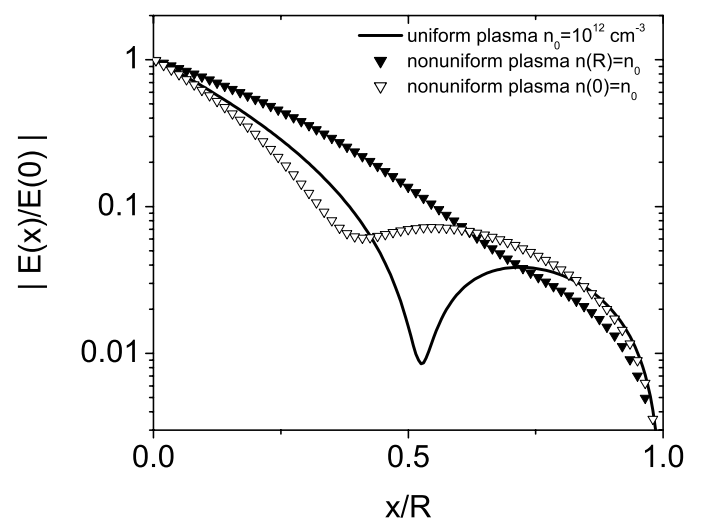

FIG. 9: 

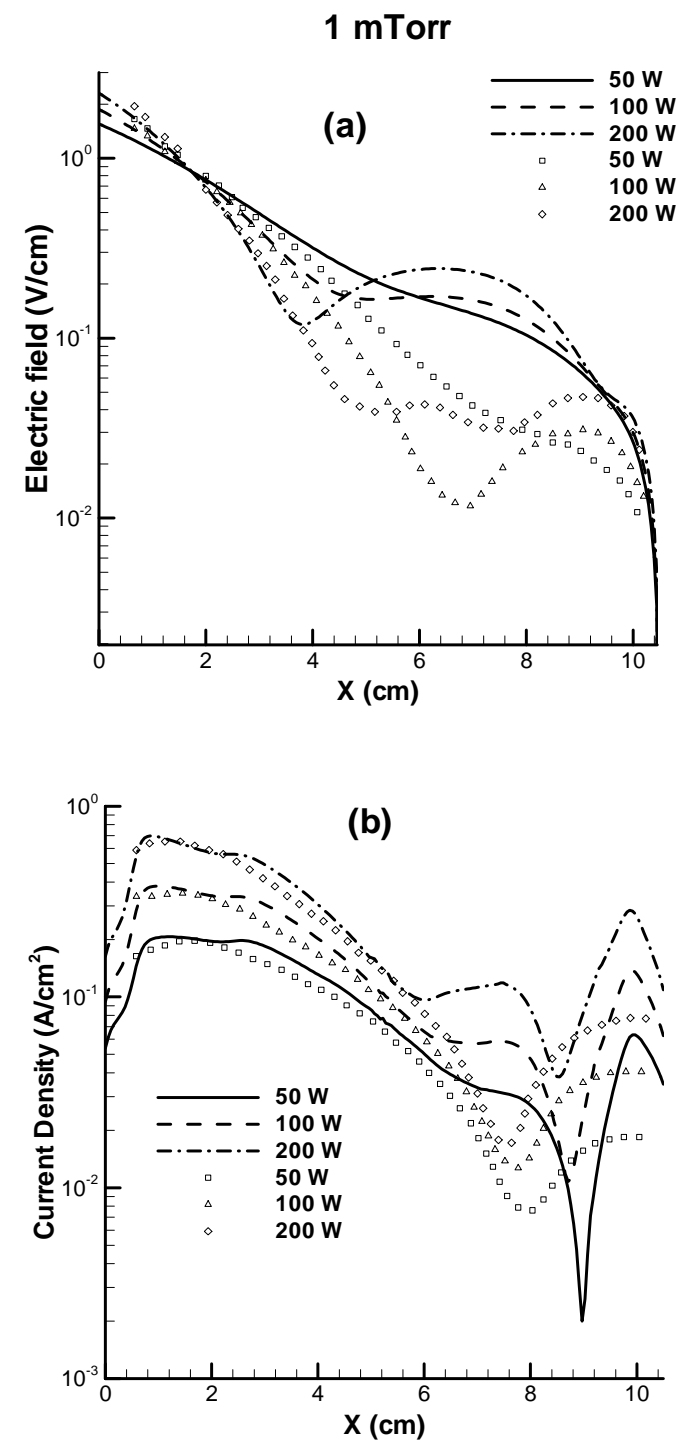

FIG. 10: 

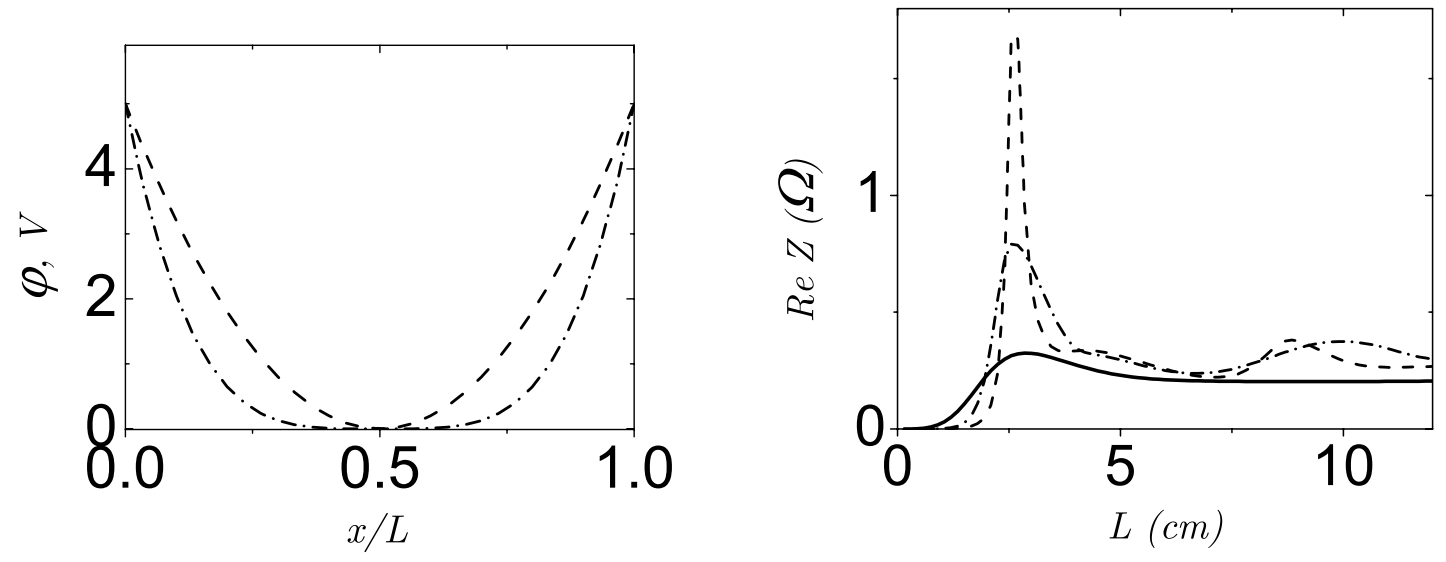

FIG. 11: 


\section{External Distribution}

Plasma Research Laboratory, Australian National University, Australia

Professor I.R. Jones, Flinders University, Australia

Professor João Canalle, Instituto de Fisica DEQ/IF - UERJ, Brazil

Mr. Gerson O. Ludwig, Instituto Nacional de Pesquisas, Brazil

Dr. P.H. Sakanaka, Instituto Fisica, Brazil

The Librarian, Culham Laboratory, England

Mrs. S.A. Hutchinson, JET Library, England

Professor M.N. Bussac, Ecole Polytechnique, France

Librarian, Max-Planck-Institut für Plasmaphysik, Germany

Jolan Moldvai, Reports Library, Hungarian Academy of Sciences, Central Research Institute for Physics, Hungary

Dr. P. Kaw, Institute for Plasma Research, India

Ms. P.J. Pathak, Librarian, Institute for Plasma Research, India

Ms. Clelia De Palo, Associazione EURATOM-ENEA, Italy

Dr. G. Grosso, Instituto di Fisica del Plasma, Italy

Librarian, Naka Fusion Research Establishment, JAERI, Japan

Library, Laboratory for Complex Energy Processes, Institute for Advanced Study, Kyoto University, Japan

Research Information Center, National Institute for Fusion Science, Japan

Dr. O. Mitarai, Kyushu Tokai University, Japan

Dr. Jiangang Li, Institute of Plasma Physics, Chinese Academy of Sciences, People's Republic of China

Professor Yuping Huo, School of Physical Science and Technology, People's Republic of China

Library, Academia Sinica, Institute of Plasma Physics, People's Republic of China

Librarian, Institute of Physics, Chinese Academy of Sciences, People's Republic of China

Dr. S. Mirnov, TRINITI, Troitsk, Russian Federation, Russia

Dr. V.S. Strelkov, Kurchatov Institute, Russian Federation, Russia

Professor Peter Lukac, Katedra Fyziky Plazmy MFF UK, Mlynska dolina F-2, Komenskeho Univerzita, SK-842 15 Bratislava, Slovakia

Dr. G.S. Lee, Korea Basic Science Institute, South Korea

Institute for Plasma Research, University of Maryland, USA

Librarian, Fusion Energy Division, Oak Ridge National Laboratory, USA

Librarian, Institute of Fusion Studies, University of Texas, USA

Librarian, Magnetic Fusion Program, Lawrence Livermore National Laboratory, USA

Library, General Atomics, USA

Plasma Physics Group, Fusion Energy Research Program, University of California at San Diego, USA

Plasma Physics Library, Columbia University, USA

Alkesh Punjabi, Center for Fusion Research and Training, Hampton University, USA

Dr. W.M. Stacey, Fusion Research Center, Georgia Institute of Technology, USA

Dr. John Willis, U.S. Department of Energy, Office of Fusion Energy Sciences, USA

Mr. Paul H. Wright, Indianapolis, Indiana, USA 
The Princeton Plasma Physics Laboratory is operated by Princeton University under contract with the U.S. Department of Energy.

\author{
Information Services \\ Princeton Plasma Physics Laboratory \\ P.O. Box 451 \\ Princeton, NJ 08543
}

Phone: 609-243-2750

Fax: 609-243-2751

e-mail: pppl_info@pppl.gov

Internet Address: http://www.pppl.gov 